\title{
Age-Related Effects and Sex Differences in Gray Matter Density, Volume, Mass, and Cortical Thickness from Childhood to Young Adulthood
}

\author{
DEfstathios D. Gennatas, ${ }^{1}$ Brian B. Avants, ${ }^{2}$ Daniel H. Wolf, ${ }^{1}$-Theodore D. Satterthwaite, ${ }^{1}$ Kosha Ruparel, ${ }^{1}$ \\ Rastko Ciric, ${ }^{1}$ Hakon Hakonarson, ${ }^{3}$ Raquel E. Gur, ${ }^{1}$ and Ruben C. Gur ${ }^{1}$ \\ ${ }^{1}$ Brain Behavior Laboratory, Department of Psychiatry, and 2Penn Image Computing and Science Laboratory, Department of Radiology, University of \\ Pennsylvania, Philadelphia, Pennsylvania 19104, and ${ }^{3}$ Center for Applied Genomics, Children's Hospital of Philadelphia, Philadelphia, Pennsylvania 19104
}

Developmental structural neuroimaging studies in humans have long described decreases in gray matter volume (GMV) and cortical thickness (CT) during adolescence. Gray matter density (GMD), a measure often assumed to be highly related to volume, has not been systematically investigated in development. We used T1 imaging data collected on the Philadelphia Neurodevelopmental Cohort to study age-related effects and sex differences in four regional gray matter measures in 1189 youths ranging in age from 8 to 23 years. Custom T1 segmentation and a novel high-resolution gray matter parcellation were used to extract GMD, GMV, gray matter mass (GMM; defined as GMD $\times$ GMV), and CT from 1625 brain regions. Nonlinear models revealed that each modality exhibits unique age-related effects and sex differences. While GMV and CT generally decrease with age, GMD increases and shows the strongest age-related effects, while GMM shows a slight decline overall. Females have lower GMV but higher GMD than males throughout the brain. Our findings suggest that GMD is a prime phenotype for the assessment of brain development and likely cognition and that periadolescent gray matter loss may be less pronounced than previously thought. This work highlights the need for combined quantitative histological MRI studies.

Key words: brain structure; cortical thickness; gray matter density; gray matter volume; MRI; T1-weighted imaging

Significance Statement

This study demonstrates that different MRI-derived gray matter measures show distinct age and sex effects and should not be considered equivalent but complementary. It is shown for the first time that gray matter density increases from childhood to young adulthood, in contrast with gray matter volume and cortical thickness, and that females, who are known to have lower gray matter volume than males, have higher density throughout the brain. A custom preprocessing pipeline and a novel high-resolution parcellation were created to analyze brain scans of 1189 youths collected as part of the Philadelphia Neurodevelopmental Cohort. A clear understanding of normal structural brain development is essential for the examination of brain-behavior relationships, the study of brain disease, and, ultimately, clinical applications of neuroimaging.

\section{Introduction}

Structural neuroimaging provides insights into the spectrum of typical and nontypical brain and neurocognitive development.

\footnotetext{
Received Nov. 17, 2016; revised March 21, 2017; accepted April 12, 2017

Author contributions: E.D.G. and B.B.A. designed research; E.D.G. performed research; E.D.G., B.B.A., D.H.W., T.D.S., K.R., R.C., H.H., R.E.G., and R.C.G. contributed unpublished reagents/analytic tools; E.D.G. analyzed data; E.D.G., D.H.W., T.D.S., R.E.G., and R.C.G. wrote the paper.

This work was supported by National Institutes of Health Grants MH-107235, MH-089983, and MH-096891, and by the Dowshen Neuroscience fund. We thank PNC participants and their families, as well as the acquisition, assessment, and recruitment team, Monica E. Calkins, Karthik Prabhakaran, Jeffrey Valdez, Marisa Riley, Jack Keefe, Nick DeLeo, Raphael Gerraty, Elliott Yodh, and Rosetta Chiavacci, and Dr. Mark Elliott, for contributing the 3D watershed code.

The authors declare no competing financial interests.

The data reported in this article has been deposited in the database of Genotypes and Phenotypes (dbGaP; www.ncbi.nlm.nih.gov/gap; Accession No. phs000607.v1.p1).
}

T1-weighted imaging is the most commonly acquired MRI sequence and offers high-resolution, low-noise images of brain structure with good tissue contrast. Several structural measures can be derived from a single T1-weighted image, including gray matter density (GMD), gray matter volume (GMV), and cortical thickness (CT). Since the early days of MRI, a large body of research has used these measures to study healthy and clinical populations. Perhaps surprisingly, confusion exists in the field as GMD, GMV, and CT are often wrongly assumed to be equivalent or highly related measures of regional gray matter quantity. GMV

Correspondence should be addressed to Efstathios D. Gennatas, Hospital of the University of Pennsylvania, 10 Gates Pavilion, Philadelphia, PA 19104. E-mail: egenn@upenn.edu.

DOI:10.1523/JNEUROSCI.3550-16.2017

Copyright $\odot 2017$ the authors $\quad 0270-6474 / 17 / 375065-09 \$ 15.00 / 0$ 
and CT are measured in cubic millimeters and millimeters, respectively. GMD, on the other hand, is a unitless, scalar measure derived from image segmentation and related to T1 signal intensity. In one form or other, gray matter abnormalities have been described in all major neurologic and psychiatric diseases. Voxelbased morphometry (VBM) analyses have suggested syndromespecific regional atrophy patterns in neurodegenerative diseases (Seeley et al., 2009). Gray matter abnormalities are widely reported in psychiatric disorders as well but paint a more complex picture (Brent et al., 2013; Bakhshi and Chance, 2015), likely reflecting both increased neuropathological heterogeneity and diagnostic variability. Much of psychopathology emerges around adolescence, a period characterized by rapid changes in behavior. Detecting and interpreting what may often be subtle and diffuse disease-related differences on top of profound and variable agerelated changes is particularly challenging. A clear, multidimensional understanding of normative structural brain development is therefore essential.

The first 2 years of life are characterized by rapid gray matter growth, which reaches its lifetime maximum at $\sim 2-3$ years of age (Matsuzawa et al., 2001; Knickmeyer et al., 2008). In contrast, myelination of white matter tracts continues well into adulthood, until the late 30s (Grydeland et al., 2013). Several developmental neuroimaging studies have described modest decreases in gray matter during adolescence using measures derived from gray matter volume and cortical thickness (Sowell et al., 2003, 2004; Gogtay et al., 2004; Shaw et al., 2008; Brain Development Cooperative Group, 2012). It should be noted that some of the early studies used the term "gray matter density" to refer to the proportion of gray matter voxels around a sphere of fixed diameter following hard segmentation of the brain (Sowell et al., 2003, 2004; Gogtay et al., 2004) and suggested that this quantity reflected local cortical thickness. Today, cortical thickness can be measured directly using automated methods and GMD usually refers to a different measure, specifically the output of soft segmentation. Unlike hard segmentation, where each voxel is labeled as "gray," "white," or "CSF" (for the common three-class case), soft segmentation creates a GMD map by assigning voxels a value between 0 and 1 , which is considered to reflect the amount of gray matter in each voxel. It is related to the T1 signal and thus to the regional proton density as well as the tissue microenvironment. To complicate things further, one of the most common measures used in the literature, and the default option in many VBM pipelines, is "modulated" gray matter density. This is equal to GMD multiplied by a scaling factor to account for volume change from the individual's native space image to the registration template. It adds to the confusion because the relative contribution of each measure is unclear and likely variable spatially and temporally (with regard to age). To date, no study has compared age-related effects on these four commonly used gray matter measures.

In this study, we used the extensive cross-sectional neuroimaging dataset collected on the Philadelphia Neurodevelopmental Cohort (PNC) to characterize age effects and sex differences on native space gray matter density, volume, and mass (defined as density $X$ volume; equivalent to modulated gray matter density), as well as cortical thickness.

\section{Materials and Methods}

Subjects and MRI acquisition. All data were collected as part of the PNC, as previously described (Satterthwaite et al., 2014). Procedures were approved by the Institutional Review Boards of the Children's Hospital of Philadelphia and the University of Pennsylvania. A total of 1189 subjects
(648 females) ranging in age from 8 to 23 years were selected from a starting total of 1601 subjects after excluding those with a neurological or psychiatric history, use of psychoactive medication, or incidental findings and those whose structural data failed quality control. The scanning of all subjects was performed on the same Siemens TIM Trio scanner at the Hospital of the University of Pennsylvania. T1-weighted imaging was obtained using a magnetization-prepared rapid acquisition gradient echo $($ MPRAGE) sequence $(\mathrm{TR}=1810 \mathrm{~ms} ; \mathrm{TE}=3.51 \mathrm{~ms} ; \mathrm{FoV}=180 \times$ $240 \mathrm{~mm}$; Resolution $=0.94 \times 0.94 \times 1.0 \mathrm{~mm})$.

MRI preprocessing. A custom T1 preprocessing pipeline was created using Advanced Normalization Tools (ANTs; https://github.com/stnava/ANTs; RRID: SCR_004757). Raw T1 volumes were first corrected for bias due to field inhomogeneity using the N4 algorithm (Tustison et al., 2010). The bias-corrected volumes were then registered to a whole-head MNI template (whole-head-to-whole-head registration). The inverse transformation was used to map the MNI brain mask to native space, which was used to isolate the brain in native space (skull stripping). The skull-stripped volume was then registered to the skull-stripped MNI template (brainto-brain registration), which results in improved registration accuracy compared with the whole-head-to-whole-head registration (Klein et al., 2010). Registrations were performed by a sequence of rigid, affine, and symmetric diffeomorphic transformations (Avants et al., 2008; Klein et al., 2009).

Gray matter density and cortical thickness estimation. MRI brain tissue segmentation is commonly guided by a set of tissue priors. Given the wide age range of our sample, we wanted to avoid using a single set of priors for all subjects or different sets of priors for different age bins. We therefore implemented an iterative process based on Atropos (Avants et al., 2011) that requires no tissue priors. On the first iteration, K-means initialization was used to derive three classes. The following two iterations used the segmentation output of the previous step for initialization. This procedure resulted in a three-class hard segmentation and a GMD map (soft segmentation) for each subject in native space (Fig. 1A). Cortical thickness was obtained using the diffeomorphic registration-based cortical thickness estimation procedure of ANTs (DiReCT, Das et al., 2009), following registration of all $\mathrm{T} 1$ images to a study-specific template. This method offers reliable CT estimation (Tustison et al., 2014) and, by providing a voxelwise measure in native volumetric space, allows the use of the same brain parcellation as the other modalities.

Quality assurance. To assess the quality of the T1 acquisition and segmentation, we calculated pairwise spatial correlations among all subjects for two sets of images: bias-field corrected, normalized T1s, and normalized GMD maps. All images whose spatial correlation was 2 SDs lower than the mean in either case were excluded $(n=56)$. A visual check confirmed the variable extent of motion artifacts in the excluded images, with those near the threshold being only minimally affected (still excluded). The motion artifact is known to significantly affect tissue segmentation and all our derived measures (Blumenthal et al., 2002; Savalia et al., 2017), and our large sample afforded us this perhaps conservative exclusion threshold.

High-resolution gray matter parcellation. Multiple methods for wholebrain parcellation have been previously proposed. Anatomical parcellations, like the AAL (Automated Analytical Labeling) atlas (Tzourio-Mazoyer et al., 2002) and the Harvard-Oxford Atlas (distributed with FSL; https://fsl.fmrib.ox.ac.uk/; RRID:SCR_002823) are based on neuroanatomy but consist of a small number of relatively large regions. Using large parcels or regions of interest runs the risk of averaging over inhomogeneous regions, resulting in signal loss. On the other hand, a number of approaches have been proposed for parcellation based on functional connectivity derived from task-free functional MRI data (also known as resting-state fMRI), but none were based on T1-weighted images. A recent approach used multimodal MRI data to create a parcellation of 180 regions in each hemisphere. As the authors note, the parcels show high variance in shape and size and consider their number to be "a lower bound, as some parcels are probably complexes of multiple areas" (Glasser et al., 2016).

Our goal was to develop a high-resolution parcellation derived from structural data where parcels are centered around GMD peaks (i.e., cortical gyri and subcortical nuclei). An age- and sex-matched subsample of 


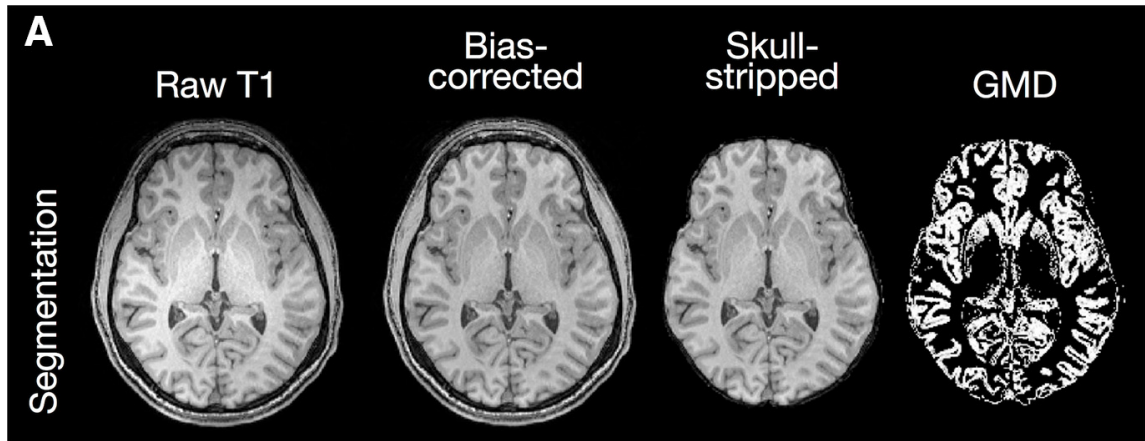

B 1 - $\mathrm{VGMD}$
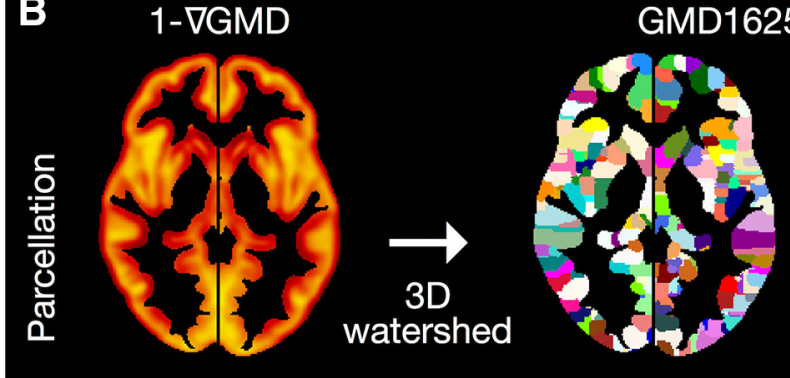

GMD1625 parcellation

Figure 1. T1 preprocessing and high-resolution gray matter parcellation. $A$, Raw T1 MPRAGE volumes were first corrected for field inhomogeneity and then skull stripped by transforming the MNI brain mask to native space. Gray matter segmentation was performed without the use of tissue priors to produce unbiased estimates of GMD. $\boldsymbol{B}$, The GMD maps of an age- and sex-balanced subsample of 240 subjects were averaged and smoothed; 1 - the gradient of the resulting image was calculated and passed to a 3D watershed algorithm, resulting in 1625 regions covering the whole-brain gray matter.

predictors can be replaced by smooth functions of themselves, offering efficient and flexible estimation of nonlinear effects. Three sets of models were fit. Full models included age and age-by-sex interaction terms represented using penalized smoothing splines with smoothing parameters selected by restricted maximum likelihood. For each modality in turn, for each gray matter parcel $p$, a model of form (1) was fit.

$\{\mathrm{GMD}, \mathrm{GMV}, \mathrm{GMM}, \mathrm{CT}\}_{\mathrm{p}} \sim \operatorname{Sex}$

$$
+s(\text { Age })+s(\text { Age } * \text { Sex })
$$

where $s()$ represents a penalized smoothing spline. The dimension of the basis used to represent the smooth terms was limited to a maximum of five in all models. Reduced models were fit to obtain accurate $p$ values for the main effects of sex and age. Specifically, model 2 omits the interaction term and was fit for each parcel to obtain $p$ values for the main effect of sex. Model 3 omits sex entirely and was therefore fit separately for each sex $s$ to obtain $p$ values for the main effect of age.

$$
\begin{aligned}
\{\mathrm{GMD}, \mathrm{GMV}, \mathrm{GMM}, \mathrm{CT}\}_{\mathrm{p}} & \sim \text { Sex } \\
& +\mathrm{s}(\text { Age })
\end{aligned}
$$

$\{\mathrm{GMD}, \mathrm{GMV}, \mathrm{GMM}, \mathrm{CT}\}_{\mathrm{s}, \mathrm{p}} \sim \mathrm{s}($ Age $)$.

the 1189 subjects was created by first splitting the initial sample by sex, then splitting each set into deciles based on its age range, and finally randomly selecting 12 subjects from each resulting subset (i.e., 12 subjects per sex per age decile), giving a total of 240 subjects. A mean image was created from the normalized, smoothed GMD maps of these subjects. To identify GMD peaks, the gradient of the mean GMD image was calculated, subtracted from 1 , and smoothed. A 3D watershed algorithm was applied on the resulting image, producing 1625 parcels covering the whole-brain gray matter (Fig. 1B).

Native space parcelwise data extraction. The GMD1625 parcellation was transformed into each subject's native space by applying the inverse of the brain-to-brain transformation (i.e., MNI-to-native space) and masked by each subject's gray matter hard segmentation. Volume and mean GMD and CT values were estimated for each parcel for each subject using the c3d utility (part of ITK-SNAP; http://www.itksnap.org/; RRID: SCR_002010). CT values were measured for 1339 of the 1625 regions, after excluding subcortical regions. To get a native space equivalent of modulated density, we derived gray matter mass (GMM) as the product of GMD and GMV. Native space analysis allows the direct measurement of GMV and the extraction of mean GMD and CT values with no interpolation. Averaging GMD and CT values within each parcel instead of applying Gaussian smoothing avoids smoothing-related artifacts, which are exaggerated in a segmented image. Cortical thickness (and therefore the gray matter segmentation) varies at $\sim 2-5 \mathrm{~mm}$, while smoothing kernels are commonly at least $8 \mathrm{~mm}$ full-width at half-maximum. In a gray matter segmentation, this results in voxel intensities being averaged with surrounding empty voxels (i.e., voxels of zero intensity), causing a drop in signal. The extent of signal drop depends on the number of surrounding empty voxels, which varies both by brain region and age. This makes intensity values from different locations incomparable and directly confounds age-related effects. Interpolation results in a similar artifact, equivalent to smoothing at the single-voxel level.

Age-related effects and sex differences. Generalized additive models (GAMs) were used to characterize age-related effects and sex differences on GMD, GMV, GMM, and CT using the mgcv package (Wood, 2011, 2012) in R (R Project for Statistical Computing; https://www.r-project.org/; RRID: SCR_001905). A GAM is similar to a generalized linear model where
Models of form 3 were also fitted at the whole-brain level, using mean GMD and CT (weighted by the number of voxels in each parcel), and total GMV and GMM, as separate dependent variables, as follows:

$\{\text { MeanGMD, MeanCT, TotalGMV, TotalGMM }\}_{s, p} \sim s($ Age $)$.

In each case, $p$ values were corrected for multiple comparisons by controlling the false discovery rate (FDR; Benjamini and Hochberg method; $q$ value $=0.05)$.

\section{Results}

Whole-brain age-related effects: gray matter density increases while volume and thickness decrease

We sought to characterize age-related effects and sex differences at the whole-brain and regional level on three independent gray matter measures, GMD, GMV, and CT, and a derived measure, $\mathrm{GMM}=\mathrm{GMD} \times \mathrm{GMV}$. At the whole-brain level, we find that total brain GMV and CT decrease from childhood to young adulthood ( $8-23$ years of age) in accordance with previous studies. In contrast, mean brain GMD increases during the same period. Whole-brain GMM shows only a modest decrease. Figure 2 shows plots of fitted values converted to relative percentages (fitted values for 8-year-old males are defined as 100\%) derived from whole-brain models (model of form 4 in Materials and Methods, Age-related effects and sex differences). Importantly, GMD is most sensitive to age, as follows: $30 \%$ and $40 \%$ of the variance of mean brain GMD is explained by age at scan time for males and females, respectively. CT follows with respective values of $30 \%$ and $24 \%$, while only $7 \%$ and $10 \%$ of the variance of total brain GMV is explained by age for males and females, respectively. 
Whole-brain sex differences: females have lower volume, higher density than males

Females were found to have lower total GMV than males, as expected by known sex differences in average head and brain size. At the same time, however, we show that females have higher mean GMD than males. Total CT was not significantly different between the two sexes in our analysis (Fig. 2).

\section{Regional variability in age-related and sex effects}

To achieve regional specificity, we created a high-resolution parcellation covering the whole-brain gray matter and consisting of 1625 regions. To summarize the large number of regional results, each of the 1625 gray matter parcels was assigned one of nine MNI labels (frontal lobe, temporal lobe, parietal lobe, occipital lobe, insula, caudate, putamen, thalamus, and cerebellum), as defined by the MNI atlas in FSL. Table 1 presents summary statistics for each measure aggregated by MNI label. GMM and GMV had the highest coefficient of variation $(\mathrm{CV}$; mean $\mathrm{CV}=26.7$ and 26.0, respectively), followed by CT (mean $\mathrm{CV}=15.6$ ). GMD showed the lowest CV (mean $\mathrm{CV}=3.7)$.

Parcelwise GAMs were fitted to investigate the regional variability of age-related and sex effects in our sample (1625 parcels for GMD, GMV, and GMM; 1339 parcels for CT). Table 2 shows the percentage of parcels with a significant main effect of age, a main effect of sex, and an age-by-sex interaction after FDR correction $(q=0.05)$, aggregated by MNI label. GMD showed significant age effects throughout the brain ( $99 \%$ of parcels in males, $99.9 \%$ in females), followed by CT ( $89 \%$ males, $88 \%$ females). GMV, on the other hand, showed significant age effects only in $52 \%$ and $65 \%$ of parcels in males and females respectively, while the numbers for GMM were $41 \%$ and $33 \%$. Sex effects were strong for GMD, GMV, and GMM. Indeed, the main effect of sex was more widespread than the main effect of age in GMV and GMM. Sex effects in CT were present in a minority of regions across the whole brain but were present in just over half of all temporal and parietal parcels. Age-by-sex interactions were virtually limited to GMD. Table 3 shows the unadjusted $p$ value corresponding to an FDR $q$ value of 0.05 and the median of unadjusted $p$ values surviving FDR correction.

To study the direction of age-related effects in each parcel, the net change from youngest to oldest was estimated by subtracting the fitted value at 8 years of age from the fitted value at 23 years of age for each modality, sex, and region and was converted to a percentage (by dividing with the fitted values at 8 years). The net change for parcels not surviving FDR correction was set to zero. GMD increased, on average, within all MNI regions, while GMV and CT decreased. Mean GMM decreased in all MNI regions other than the temporal lobe, insula, and cerebellum. The bilateral insula stands out, showing the highest increase in GMD and GMM of all the MNI regions. To characterize the sensitivity to age of each parcel, we examined the adjusted $R^{2}$ value of each model, denoting the percentage of variance of the regional values of each modality explained by age. Table 4 lists $R^{2}$ values and the percentage change for each modality. Averaging by MNI label masks the variability within each label; for example, a lobe may on average decrease in volume, but some parcels within it may increase. For this reason, Table 4 includes numbers of individual parcels with a net positive and a net negative change from 8 to 23 years of age within each MNI label. Brain slices mapping the net change and $R^{2}$ value for each modality are shown in Figure 3 and are available in interactive format on-line (https://egenn.github.io/gmdvdev).

Figure 4 helps describe how development modulates sex effects by plotting the average difference of male and female fitted values per modality per MNI region by age from 8 to 23 years of age. Males and females have no differences in GMD at 8 years of age, but females start to lead soon thereafter throughout the brain. Males have higher GMV and GMM on average in each MNI region throughout this age range. Only CT shows a change in the direction of sex differences with age. Males have higher CT values in bilateral insula until $\sim 12$ years of age and in frontal and occipital lobes until age 15 years of age, at which points the effect reverses, leading to a female advantage. In most cases, the sex differences have a nonlinear relationship with age.

\section{GMM largely resembles GMV, not GMD}

We defined GMM as the product of GMD and GMV to study a native-space equivalent of modulated density, a very popular measure in structural neuroimaging studies. GMM showed agerelated effects that, for the most part, closely paralleled those of GMV (Fig. 5). This is probably because GMV has much higher variance than GMD (Table 1) and consequently contributes much more to the variance in GMM explained by age than GMD does. Despite this, three MNI regions showed, on average, opposite direction of net change in GMM than in GMV (i.e., an increase instead of a decrease). This was observed in both males and females, in descending order of magnitude, in the insula, the temporal lobe, and the cerebellum (Table 4).

\section{Development modulates intermodal relationships among structural measures}

To summarize the differences in age-related effects among the four measures with a single quantity, we calculated pairwise correlation coefficients of fitted values for our sample's age range (8-23 years old; Fig. 5, top row). Spearman correlation was used as most fitted values are nonlinear. As expected from the results above, GMD was negatively correlated with GMV and CT throughout the brain and cortex respectively. GMM was posi- 
Table 1. Summary statistics of regional gray matter measures averaged by MNI label

\begin{tabular}{|c|c|c|c|c|c|c|c|c|c|c|c|c|c|}
\hline \multirow[b]{2}{*}{ MNI label } & \multicolumn{3}{|l|}{ GMD } & \multicolumn{3}{|l|}{ GMV } & \multicolumn{3}{|l|}{ GMM } & \multicolumn{3}{|l|}{ CT } & \multirow[b]{2}{*}{$N$ total } \\
\hline & Mean & SD & $\mathrm{CV}$ & Mean & SD & $\mathrm{CV}$ & Mean & SD & $\mathrm{CV}$ & Mean & SD & $\mathrm{CV}$ & \\
\hline Frontal & 0.82 & 0.03 & 3.96 & 359.75 & 99.73 & 27.72 & 295.18 & 83.19 & 28.18 & 3.57 & 0.53 & 14.82 & 514 \\
\hline Temporal & 0.82 & 0.03 & 3.77 & 352.64 & 94.07 & 26.68 & 290.09 & 79.32 & 27.34 & 4.03 & 0.60 & 14.86 & 319 \\
\hline Parietal & 0.82 & 0.03 & 3.79 & 385.09 & 114.59 & 29.76 & 317.25 & 95.28 & 30.03 & 3.34 & 0.53 & 15.88 & 294 \\
\hline Occipital & 0.80 & 0.03 & 3.54 & 290.55 & 95.01 & 32.70 & 235.55 & 78.16 & 33.18 & 3.27 & 0.62 & 18.86 & 190 \\
\hline Insula & 0.83 & 0.03 & 3.76 & 578.78 & 106.87 & 18.47 & 478.39 & 88.37 & 18.47 & 4.53 & 0.61 & 13.49 & 22 \\
\hline Caudate & 0.84 & 0.03 & 3.20 & 374.18 & 61.72 & 16.50 & 313.19 & 52.72 & 16.83 & & & & 25 \\
\hline Putamen & 0.76 & 0.03 & 4.12 & 317.26 & 92.32 & 29.10 & 242.50 & 74.38 & 30.67 & & & & 23 \\
\hline Thalamus & 0.77 & 0.03 & 3.38 & 316.30 & 80.05 & 25.31 & 246.39 & 64.68 & 26.25 & & & & 25 \\
\hline Cerebellum & 0.80 & 0.03 & 3.74 & 340.28 & 94.78 & 27.85 & 273.04 & 78.83 & 28.87 & & & & 213 \\
\hline
\end{tabular}

$N$ total, total number of parcels within MNI label (of 1625).

Table 2. Generalized Additive Models: Main effects and interaction by MNI label: percentage of parcels with significant effects after FDR correction for multiple comparisons

\begin{tabular}{|c|c|c|c|c|c|c|c|c|c|c|c|c|c|c|c|c|c|}
\hline \multirow[b]{3}{*}{ MNI label } & \multicolumn{8}{|c|}{ Main effect of age } & & & & & & & & & \\
\hline & \multicolumn{2}{|l|}{ GMD } & \multicolumn{2}{|l|}{ GMV } & \multicolumn{2}{|c|}{ GMM } & \multicolumn{2}{|l|}{$\mathrm{CT}$} & \multicolumn{4}{|c|}{ Main effect of sex } & \multicolumn{4}{|c|}{ Age by sex interaction } & \multirow[b]{2}{*}{$N$ total } \\
\hline & $M$ & $\mathrm{~F}$ & $M$ & $\mathrm{~F}$ & M & $\mathrm{F}$ & M & $\mathrm{F}$ & GMD & GMV & GMM & $\mathrm{CT}$ & GMD & GMV & GMM & $\mathrm{CT}$ & \\
\hline Frontal & 99 & 100 & 44 & 67 & 35 & 27 & 92 & 91 & 100 & 88 & 81 & 24 & 79 & 4 & 7 & 1 & 514 \\
\hline Temporal & 100 & 100 & 43 & 65 & 35 & 34 & 75 & 78 & 99 & 95 & 91 & 56 & 94 & 2 & 2 & 1 & 319 \\
\hline Parietal & 98 & 100 & 64 & 74 & 46 & 38 & 96 & 93 & 99 & 84 & 80 & 52 & 91 & 4 & 6 & 4 & 294 \\
\hline Occipital & 99 & 100 & 69 & 47 & 49 & 18 & 92 & 84 & 96 & 74 & 78 & 44 & 92 & 8 & 16 & 15 & 190 \\
\hline Insula & 100 & 100 & 14 & 59 & 32 & 32 & 100 & 100 & 100 & 100 & 100 & 14 & 45 & 0 & 0 & 0 & 22 \\
\hline Caudate & 96 & 100 & 24 & 92 & 20 & 32 & & & 100 & 96 & 96 & & 44 & 4 & 4 & & 25 \\
\hline Putamen & 83 & 91 & 100 & 100 & 100 & 96 & & & 70 & 91 & 87 & & 61 & 9 & 13 & & 23 \\
\hline Thalamus & 100 & 100 & 64 & 76 & 48 & 64 & & & 88 & 96 & 96 & & 32 & 8 & 8 & & 25 \\
\hline Cerebellum & 100 & 100 & 51 & 54 & 48 & 46 & & & 79 & 87 & 85 & & 66 & 2 & 4 & & 213 \\
\hline
\end{tabular}

F, Female; M, Male.

Table 3. Generalized additive models: FDR threshold and median $p$ values

\begin{tabular}{ccc}
\hline & Threshold & Median \\
\hline $\begin{array}{l}\text { Main effect of age } \\
\text { GMD }\end{array}$ & & \\
M & & \\
F & 0.0494 & $2.005 \mathrm{E}-24$ \\
GMV & 0.0500 & $3.096 \mathrm{E}-47$ \\
M & & \\
F & 0.0276 & $2.359 \mathrm{E}-04$ \\
GMM & 0.0337 & $2.516 \mathrm{E}-04$ \\
M & & \\
F & 0.0227 & $2.612 \mathrm{E}-04$ \\
CT & 0.0197 & $2.516 \mathrm{E}-04$ \\
M & & \\
F & 0.0448 & $2.195 \mathrm{E}-09$ \\
Main effect of sex & 0.0442 & $2.989 \mathrm{E}-10$ \\
GMD & & \\
GMV & 0.0480 & $1.437 \mathrm{E}-12$ \\
GMM & 0.0446 & $1.448 \mathrm{E}-09$ \\
CT & 0.0427 & $9.395 \mathrm{E}-08$ \\
Age by sex interaction & & \\
GMD & $0.327 \mathrm{E}-04$ \\
GMV & & $6.223 \mathrm{E}-04$ \\
GMM & 0.0414 & $3.427 \mathrm{E}-04$ \\
CT & 0.0029 & $6.872 \mathrm{E}-04$ \\
\hline
\end{tabular}

F, Female; M, Male; Threshold, unadjusted $p$ value corresponding to FDR $q$ value of 0.05 ; median, median of unadjusted $p$ values surviving FDR correction.

tively correlated with GMV and CT in all MNI regions, on average, with the exception of the insula. Looking at the same pairwise correlations among the residuals (i.e., after removing the effect of age), we see that most correlations are positive, with the notable exception of the density and thickness pair in the insula (Fig. 5, bottom row). Intermodal correlations of residuals help to suggest what relationships may look like in the absence of an age effect but are no substitute for directly examining separate age bins, which should ideally extend across the life span.

\section{Discussion}

Despite extensive use of different MRI-derived gray matter measures in the literature, very few attempts have been made to directly compare them or their developmental patterns, and they are often wrongly assumed to be equivalent. This study shows distinct age-related effects and sex differences on whole-brain and regional measures of GMD, GMV, GMM $(\mathrm{GMM}=\mathrm{GMD} \times$ GMV), and CT in a cross-sectional dataset of 1189 youths ranging in age from 8 to 23 years drawn from the PNC. A custom T1 preprocessing pipeline and a novel high-resolution gray matter parcellation were created to produce unbiased gray matter segmentations without use of priors and to extract native space measures without any interpolation or smoothing. Our findings partly challenge the widely held, though vague, view that "gray matter declines" from childhood to young adulthood and provide a more complete description of developmental gray matter differences.

\section{Not all gray matter declines in adolescence}

Mean brain GMD increases from childhood to young adulthood, while total brain GMV and mean CT decrease. Total GMM only shows a slight decrease from 8 to 23 years of age, suggesting that an increase in density may partly counter a decrease in volume. Regionally, GMD increases virtually throughout the brain. GMV, on the other hand, decreases on average in all lobes and subcortical regions, but there are parcels within those broad regions whose volume increases, particularly in frontotemporal cortex. 
Table 4. Net percentage change from 8 to 23 years of age and variance explained by MNI label

\begin{tabular}{|c|c|c|c|c|c|c|c|c|c|c|c|c|c|c|c|c|c|}
\hline \multirow[b]{2}{*}{ MNI label } & \multicolumn{4}{|l|}{ GMD } & \multicolumn{4}{|l|}{ GMV } & \multicolumn{4}{|l|}{ GMM } & \multicolumn{4}{|l|}{ CT } & \multirow[b]{2}{*}{$N$ total } \\
\hline & $R^{2}$ & $P c t$ & $N \uparrow$ & $N \downarrow$ & $R^{2}$ & Pct & $N \uparrow$ & $N \downarrow$ & $R^{2}$ & Pct & $N \uparrow$ & $N \downarrow$ & $R^{2}$ & $P c t$ & $N \uparrow$ & $N \downarrow$ & \\
\hline \multicolumn{18}{|l|}{ Males } \\
\hline Frontal & 0.181 & 5.48 & 507 & 1 & 0.015 & -5.34 & 35 & 193 & 0.012 & -1.37 & 66 & 116 & 0.088 & -12.75 & 23 & 450 & 514 \\
\hline Temporal & 0.207 & 5.79 & 319 & 0 & 0.013 & -2.69 & 45 & 92 & 0.015 & 1.70 & 69 & 44 & 0.027 & -5.43 & 30 & 209 & 319 \\
\hline Parietal & 0.168 & 4.99 & 288 & 0 & 0.021 & -10.17 & 12 & 176 & 0.015 & -5.22 & 24 & 112 & 0.097 & -15.87 & 4 & 278 & 294 \\
\hline Occipital & 0.170 & 5.09 & 189 & 0 & 0.026 & -11.02 & 13 & 118 & 0.018 & -6.33 & 15 & 78 & 0.063 & -14.11 & 5 & 169 & 190 \\
\hline Insula & 0.393 & 8.51 & 22 & 0 & 0.002 & -1.04 & 0 & 3 & 0.008 & 3.63 & 7 & 0 & 0.140 & -17.49 & 0 & 22 & 22 \\
\hline Caudate & 0.202 & 4.78 & 24 & 0 & 0.009 & -3.15 & 0 & 6 & 0.006 & -0.27 & 3 & 2 & & & & & 25 \\
\hline Putamen & 0.058 & 2.60 & 18 & 1 & 0.122 & -28.70 & 0 & 23 & 0.097 & -26.76 & 0 & 23 & & & & & 23 \\
\hline Thalamus & 0.222 & 5.59 & 25 & 0 & 0.034 & -10.60 & 2 & 14 & 0.028 & -5.92 & 3 & 9 & & & & & 25 \\
\hline Cerebellum & 0.216 & 5.78 & 213 & 0 & 0.028 & -3.02 & 32 & 76 & 0.025 & 0.91 & 50 & 53 & & & & & 213 \\
\hline \multicolumn{18}{|l|}{ Females } \\
\hline Frontal & 0.263 & 8.21 & 514 & 0 & 0.017 & -8.68 & 22 & 321 & 0.007 & -0.84 & 40 & 100 & 0.075 & -11.15 & 25 & 444 & 514 \\
\hline Temporal & 0.296 & 8.51 & 319 & 0 & 0.016 & -6.48 & 40 & 167 & 0.013 & 1.89 & 60 & 47 & 0.026 & -5.27 & 36 & 214 & 319 \\
\hline Parietal & 0.293 & 8.41 & 294 & 0 & 0.022 & -12.16 & 6 & 213 & 0.009 & -3.36 & 20 & 93 & 0.092 & -14.86 & 8 & 266 & 294 \\
\hline Occipital & 0.262 & 7.66 & 190 & 0 & 0.012 & -6.65 & 5 & 85 & 0.004 & -1.48 & 7 & 27 & 0.045 & -10.81 & 15 & 146 & 190 \\
\hline Insula & 0.430 & 10.39 & 22 & 0 & 0.010 & -5.42 & 0 & 13 & 0.006 & 2.92 & 7 & 0 & 0.095 & -15.16 & 0 & 22 & 22 \\
\hline Caudate & 0.243 & 6.36 & 25 & 0 & 0.032 & -10.65 & 0 & 23 & 0.006 & -2.97 & 0 & 8 & & & & & 25 \\
\hline Putamen & 0.107 & 5.24 & 21 & 0 & 0.125 & -26.15 & 0 & 23 & 0.091 & -21.99 & 0 & 22 & & & & & 23 \\
\hline Thalamus & 0.256 & 6.61 & 25 & 0 & 0.067 & -19.71 & 0 & 19 & 0.046 & -14.91 & 2 & 14 & & & & & 25 \\
\hline Cerebellum & 0.259 & 8.16 & 213 & 0 & 0.021 & -3.70 & 26 & 88 & 0.016 & 2.18 & 49 & 48 & & & & & 213 \\
\hline
\end{tabular}

Pct, Percentage change of fitted values from 8 to 23 years of age; $N \uparrow$, number of parcels with positive net change (increase); $N \downarrow$, number of parcels with negative net change (decrease; only parcels that survived FDR correction have been considered).

Future work will determine whether areas that expand during adolescence despite an overall decline in volume support the enhancement of specific neurocognitive functions.

Age-related effects and sex differences in density may help understand cognitive abilities

We know that higher GMV correlates with higher neurocognitive performance in adults (Gur et al., 1999; McDaniel, 2005), which gives rise to the following two apparent paradoxes: (1) adolescence is characterized by a sharp rise in neurocognitive performance (Gur and Gur, 2016), despite a decline in GMV; and (2) there are no significant sex differences in general intelligence (Halpern et al., 2007), despite a male advantage in GMV. Our results suggest that an age-associated volume decrease might be compensated for by increasing gray matter density during adolescence, and lower volume in females might be compensated for by higher density throughout the brain.

\section{Biological basis of structural MR} measures: the need for large-scale, quantitative histological MRI studies The above findings beg the following question: what do GMD and the other gray matter measures mean in terms of biology? Multiple studies have shown that the $\mathrm{T} 1$ signal is sensitive to myelin and iron content, whose distributions overlap significantly within cortical gray matter (Stüber et al., 2014). Surprisingly, only a few attempts have been made to quantify
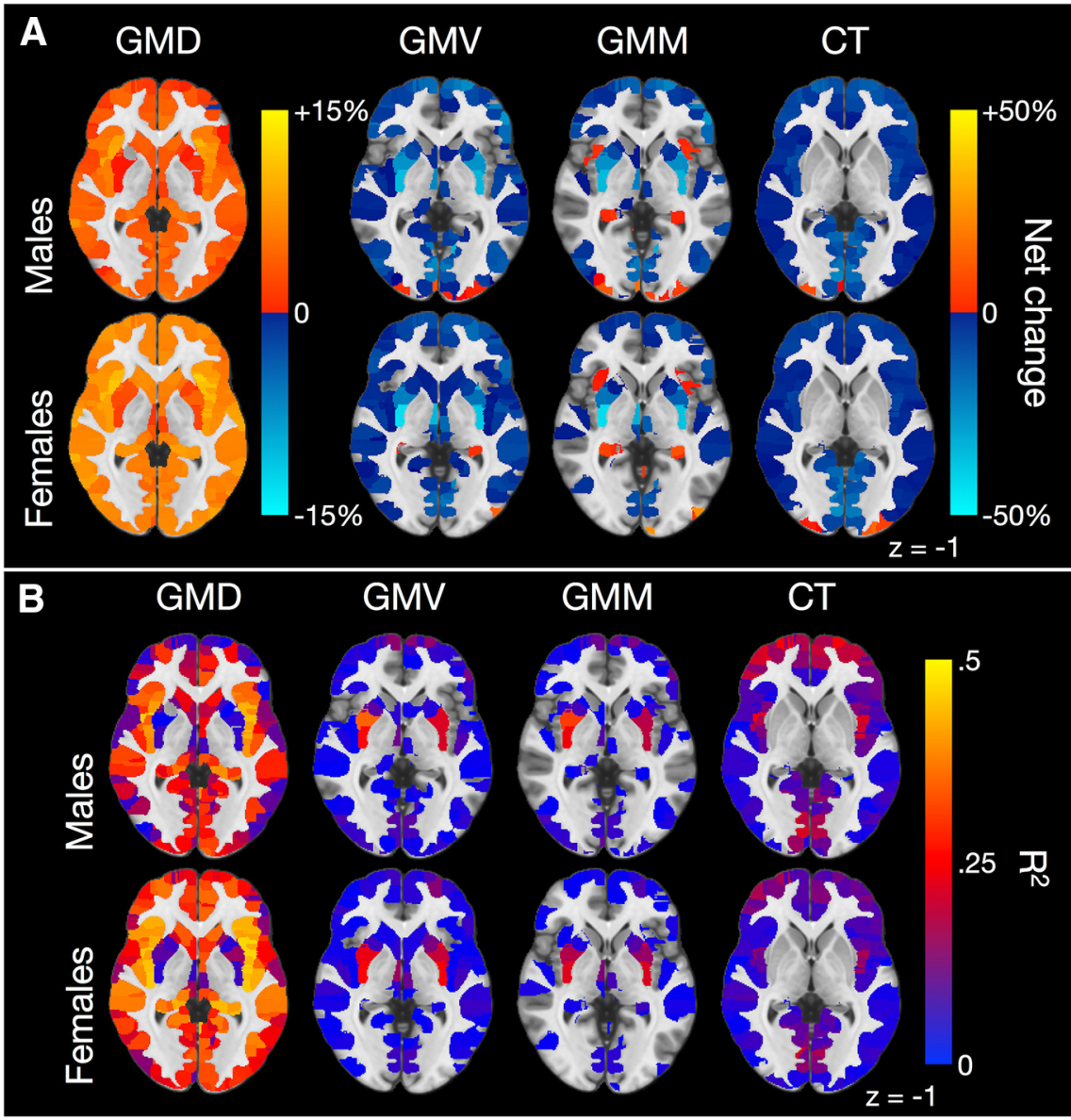

Figure 3. Percentage net change and variance explained by sex and modality. $\boldsymbol{A}$, For each parcel, the percentage net change was calculated as follows: (fitted value at 23 - fitted value at 8)/(fitted value at 8 ) $\times 100 \%$. GMD increased virtually throughout the brain, while the other modalities show mostly decreases. Females showed a greater increase in density than males throughout the brain. $\boldsymbol{B}$, Percentage variance of each measure explained by age. GMD showed the highest $R^{2}$ values, followed by CT. High bilateral symmetry on all maps suggests biological plausibility. Interactive movies including all axial slices in this figure are available on-line at https://egenn.github.io/gmdvdev. 

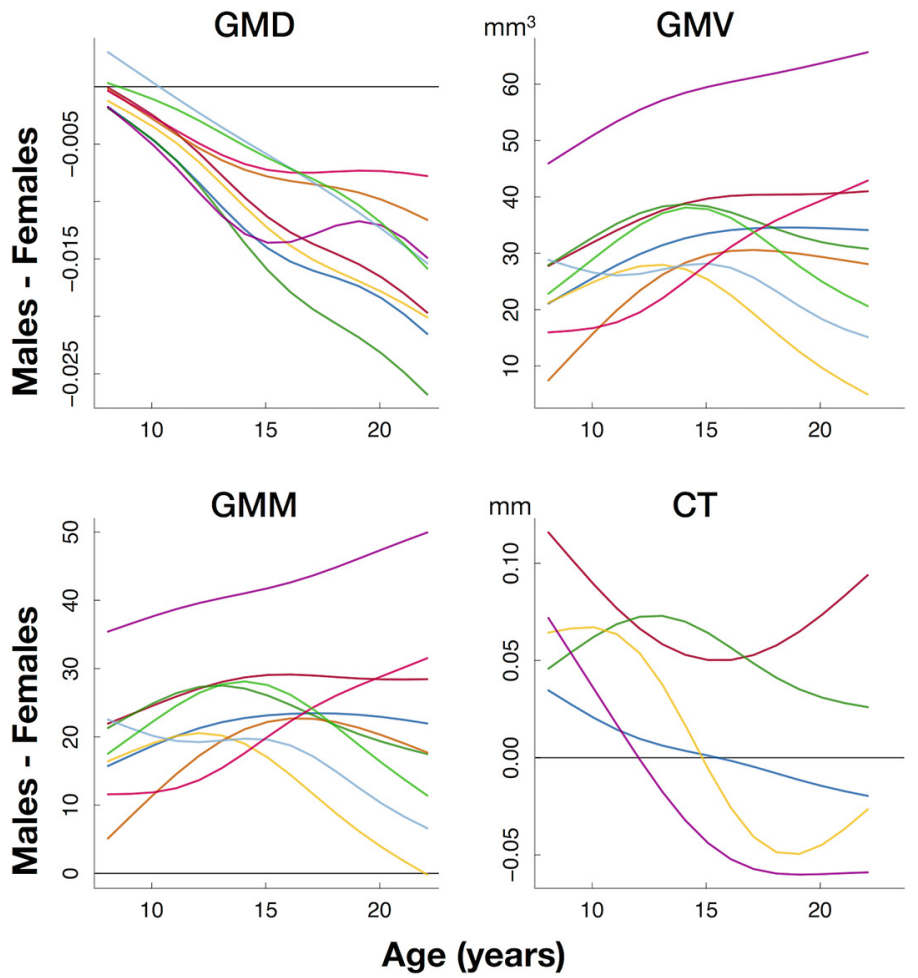

Figure 4. Sex differences by modality by MNI label against age. The difference of male and female fitted values for each modality for each MNI label was calculated at each year from 8 to 23 years of age. This plot highlights qualitatively how sex differences vary with age, in most cases in a nonlinear fashion (a constant sex difference in any measure would appear as a horizontal line). Note that only in (T the direction of the difference changes in frontal and occipital lobes as well as the bilateral insula from a male to a female advantage. the relationships among histological features and MRI-derived structural measures. A large number of studies using T1-weighted imaging to quantify gray matter have focused on neurodegeneration. Such diseases result in neuronal loss, which causes direct decreases in all gray matter measures. This may partly explain the confusion that has led to these measures often being considered highly correlated or equivalent, and even grouped together in meta-analyses (e.g., see Shao et al., 2014). However, in the context of normal brain structure, or in brain disease without extensive neuronal loss, including most psychiatric disorders, regional and global variation in different gray matter measures may be less correlated, even anticorrelated, as seen here between GMD and GMV. We expect that MRI-derived gray matter measures are differentially determined by a set of histological factors, including neuronal and glial number and size, dendritic arborization, the number of axonal projections, and the extent of myelination. Their effects will vary by age, brain region, and cortical layer.

In adolescence, the MRI-estimated decline in gray matter volume is generally attributed to a combination of synaptic pruning of exuberant connections, a regressive event, and increasing myelina-

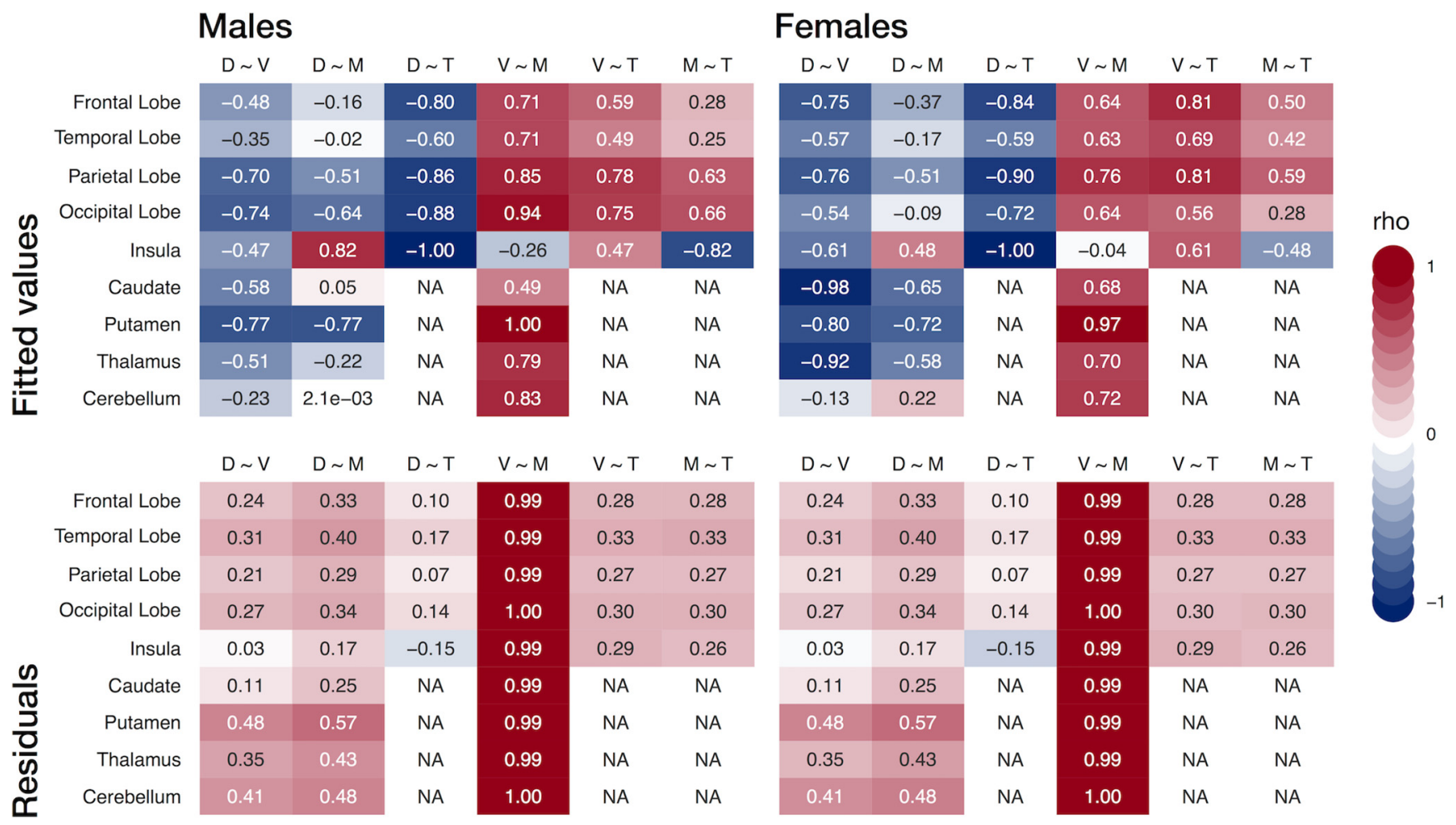

Figure 5. Intermodal correlations averaged by MNI label. Pairwise spearman correlations (rho) were estimated between the fitted values of model 3 (top row) of all gray matter measures to summarize the similarity of age-related effects among modalities and between their residuals (bottom row). Brain slices with these results are available on-line at https://egenn.github.io/ gmdvdev/imcor.html. D, Gray matter density; V, gray matter volume; $M$, gray matter mass; T, cortical thickness. 
tion, a progressive event, both of which are essential aspects of normal development (Stiles and Jernigan, 2010). While pruning results in a direct reduction in neuropil, myelination may have multiple direct and indirect effects on T1-based gray matter quantification. White matter myelination and expansion may result in a physical outward shift of the gray matter-white matter boundary, causing gray matter to compact and leading to decreases in GMV and CT and increases in GMD. Alternatively, myelination near the gray matter-white matter border may increase signal intensity in voxels nearest the border enough to switch their classification from gray to white, which would lead to a reduction of volume and thickness measurements but have no effect on density, since these voxels would now be excluded from any gray matter parcels. At the same time, cortical gray matter also contains substantial amounts of myelinated fibers with significant regional variability (Nieuwenhuys, 2013), and intracortical myelin also increases during adolescence (Grydeland et al., 2013). Increasing cortical myelination would lead to a decrease in estimated GMD, which means that GMD increases reported in this study are possibly underestimates. Rabinowicz et al. (2009) performed stereologic morphometry in six males and five females ranging in age from 12 to 24 years and reported significantly higher neuronal densities and neuronal number estimates in males than in females, but no sex differences in cortical thickness, suggesting higher neuropil mass/increased neuronal processes in the female cortex, which might explain our findings of higher GMD in females.

We chose to compare four different measures of regional gray matter in volumetric space. Other morphometric and morphological measures like cortical surface area and gyrification index can also be derived from T1 images in surface space analyses. The limited positive correlation that we found between GMV and CT age-related effects is probably explained by independent changes in surface area and gyrification (Raznahan et al., 2011). While the majority of brain regions show significant sex effects on GMV, as expected, a minority of regions showed a significant sex effect on CT. Considering that gray matter volume approximately equals surface area times cortical thickness, we expect surface area to exhibit more extensive sex differences than thickness. We limited our analysis to volumetric space measures to use the same parcellation for each measure and avoid the extra resampling and registration errors introduced in the conversion between the two spaces (Klein et al., 2010). For the same reason, care must be taken when comparing volumetric and surface space analyses.

Given the important gaps in our understanding of the links between biology and imaging, it is crucial to design large-scale, combined MRI and histological quantification studies to fully characterize the neurobiological basis of raw MRI signals and derived measures. Biophysical modeling of MR-derived measures will enable accurate noninvasive in vivo prediction of histological features (Stiles and Jernigan, 2010). This will be crucial in elevating the potential of neuroimaging in the investigation of nervous system physiology and pathology, disease diagnosis, and treatment monitoring.

\section{Limitations and implication for future work: phenotypes of structural brain development and links to cognition}

The cross-sectional design of this study was its main limitation. Ongoing longitudinal studies will provide true measures of developmental change and allow the analysis of interindividual differences in development. Future studies would also benefit from the inclusion of more MRI modalities. New diffusion-weighted MRI techniques like neurite orientation dispersion and density imaging may provide rich information on gray matter structure and complement T1 and T2 signals (Zhang et al., 2012). Histological morphometry has shown cortical layer- and type-specific changes in neuronal cell bodies (Rabinowicz et al., 2009), which cannot be resolved with today's common MRI sequences, but this may be possible in the future. We must note that while different segmentation software uses similar methods for GMD estimation, results are dependent on parameter selection. Correlation with histology will also help to guide these choices and optimize pipelines to produce measures with maximal biological interpretability.

Our results demonstrate that GMD, GMV, and CT must be considered distinct and complementary. They also further emphasize the need for nonlinear modeling and accounting for sex differences. We found that GMD and CT are most sensitive to age, which makes them prime candidate biomarkers of brain development. In contrast, modulated density or GMM may not be very informative in a developmental context, and it is best to consider GMD and GMV independently. We also show that intermodal relationships change with age, which further emphasizes that neuroimaging findings should not be generalized from one age period to another. We have previously shown that structural covariance networks develop during childhood to mirror adult functional intrinsic connectivity networks (Zielinski et al., 2010). Ongoing work aims to identify how different structural measures can be best applied to study cognition and disease.

As we advance from group-level to individual-level studies, from unimodal to multimodal analyses, and from descriptive to predictive models with the aim of integrating neuroimaging into clinical practice, it is essential to make the best use of all available data. The first step is to understand available measures and the relationships among them. Development is a critical dimension on which these relationships may vary and adds to the challenge and the importance of this task.

\section{Notes}

Supplemental material for this article is available at https://egenn.github. io/gmdvdev. Gray matter development: net percentage change, $R^{2}$, and Intermodal Correlations. This material has not been peer reviewed.

\section{References}

Avants BB, Epstein CL, Grossman M, Gee JC (2008) Symmetric diffeomorphic image registration with cross-correlation: evaluating automated labeling of elderly and neurodegenerative brain. Med Image Anal 12:2641. CrossRef Medline

Avants BB, Tustison NJ, Wu J, Cook PA, Gee JC (2011) An open source multivariate framework for n-tissue segmentation with evaluation on public data. Neuroinformatics 9:381-400. CrossRef Medline

Bakhshi K, Chance SA (2015) The neuropathology of schizophrenia: a selective review of past studies and emerging themes in brain structure and cytoarchitecture. Neuroscience 303:82-102. CrossRef Medline

Blumenthal JD, Zijdenbos A, Molloy E, Giedd JN (2002) Motion artifact in magnetic resonance imaging: implications for automated analysis. Neuroimage 16:89-92. CrossRef Medline

Brain Development Cooperative Group (2012) Total and regional brain volumes in a population-based normative sample from 4 to 18 years: the NIH MRI Study of Normal Brain Development. Cereb Cortex 22:1-12. CrossRef Medline

Brent BK, Thermenos HW, Keshavan MS, Seidman LJ (2013) Gray matter alterations in schizophrenia high-risk youth and early-onset schizophrenia: a review of structural MRI findings. Child Adolesc Psychiatr Clin N Am 22:689-714. CrossRef Medline

Das SR, Avants BB, Grossman M, Gee JC (2009) Registration based cortical thickness measurement. Neuroimage 45:867-879. CrossRef Medline

Glasser MF, Coalson TS, Robinson EC, Hacker CD, Harwell J, Yacoub E, Ugurbil K, Andersson J, Beckmann CF, Jenkinson M, Smith SM, Van 
Essen DC (2016) A multi-modal parcellation of human cerebral cortex. Nature 536:171-178. CrossRef Medline

Gogtay N, Giedd JN, Lusk L, Hayashi KM, Greenstein D, Vaituzis AC, Nugent TF 3rd, Herman DH, Clasen LS, Toga AW, Rapoport JL, Thompson PM (2004) Dynamic mapping of human cortical development during childhood through early adulthood. Proc Natl Acad Sci U S A 101:8174-8179. CrossRef Medline

Grydeland H, Walhovd KB, Tamnes CK, Westlye LT, Fjell AM (2013) Intracortical myelin links with performance variability across the human lifespan: results from T1- and T2-weighted MRI myelin mapping and diffusion tensor imaging. J Neurosci 33:18618-18630. CrossRef Medline

Gur RC, Turetsky BI, Matsui M, Yan M, Bilker W, Hughett P, Gur RE (1999) Sex differences in brain gray and white matter in healthy young adults: correlations with cognitive performance. J Neurosci 19:4065-4072. Medline

Gur RE, Gur RC (2016) Sex differences in brain and behavior in adolescence: findings from the Philadelphia Neurodevelopmental Cohort. Neurosci Biobehav Rev 70:159-170. CrossRef Medline

Halpern DF, Benbow CP, Geary DC, Gur RC, Hyde JS, Gernsbacher MA (2007) The science of sex differences in science and mathematics. Psychol Sci Public Interest 8:1-51. CrossRef Medline

Klein A, Andersson J, Ardekani BA, Ashburner J, Avants B, Chiang MC, Christensen GE, Collins DL, Gee J, Hellier P, Song JH, Jenkinson M, Lepage C, Rueckert D, Thompson P, Vercauteren T, Woods RP, Mann JJ, Parsey RV (2009) Evaluation of 14 nonlinear deformation algorithms applied to human brain MRI registration. Neuroimage 46:786-802. CrossRef Medline

Klein A, Ghosh SS, Avants B, Yeo BT, Fischl B, Ardekani B, Gee JC, Mann JJ, Parsey RV (2010) Evaluation of volume-based and surface-based brain image registration methods. Neuroimage 51:214-220. CrossRef Medline

Knickmeyer RC, Gouttard S, Kang C, Evans D, Wilber K, Smith JK, Hamer RM, Lin W, Gerig G, Gilmore JH (2008) A structural MRI study of human brain development from birth to 2 years. J Neurosci 28:1217612182. CrossRef Medline

Matsuzawa J, Matsui M, Konishi T, Noguchi K, Gur RC, Bilker W, Miyawaki $\mathrm{T}$ (2001) Age-related volumetric changes of brain gray and white matter in healthy infants and children. Cereb Cortex 11:335-342. CrossRef Medline

McDaniel M (2005) Big-brained people are smarter: a meta-analysis of the relationship between in vivo brain volume and intelligence. Intelligence 33:337-346. CrossRef

Nieuwenhuys R (2013) The myeloarchitectonic studies on the human cerebral cortex of the Vogt-Vogt school, and their significance for the interpretation of functional neuroimaging data. Brain Struct Funct 218:303-352. CrossRef Medline

Rabinowicz T, Petetot JM, Khoury JC, de Courten-Myers GM (2009) Neocortical maturation during adolescence: change in neuronal soma dimension. Brain Cogn 69:328-336. CrossRef Medline

Raznahan A, Shaw P, Lalonde F, Stockman M, Wallace GL, Greenstein D, Clasen L, Gogtay N, Giedd JN (2011) How does your cortex grow? J Neurosci 31:7174-7177. CrossRef Medline

Satterthwaite TD, Elliott MA, Ruparel K, Loughead J, Prabhakaran K, Calkins ME, Hopson R, Jackson C, Keefe J, Riley M, Mentch FD, Sleiman P,
Verma R, Davatzikos C, Hakonarson H, Gur RC, Gur RE (2014) Neuroimaging of the Philadelphia Neurodevelopmental Cohort. NeuroImage 86:544-553. CrossRef

Savalia NK, Agres PF, Chan MY, Feczko EJ, Kennedy KM, Wig GS (2017) Motion-related artifacts in structural brain images revealed with independent estimates of in-scanner head motion. Hum Brain Mapp 38: 472-492. CrossRef Medline

Seeley WW, Crawford RK, Zhou J, Miller BL, Greicius MD (2009) Neurodegenerative diseases target large-scale human brain networks. Neuron 62:42-52. CrossRef Medline

Shao N, Yang J, Li J, Shang HF (2014) Voxelwise meta-analysis of gray matter anomalies in progressive supranuclear palsy and Parkinson's disease using anatomic likelihood estimation. Front Hum Neurosci 8:63. CrossRef Medline

Shaw P, Kabani NJ, Lerch JP, Eckstrand K, Lenroot R, Gogtay N, Greenstein D, Clasen L, Evans A, Rapoport JL, Giedd JN, Wise SP (2008) Neurodevelopmental trajectories of the human cerebral cortex. J Neurosci 28 : 3586-3594. CrossRef Medline

Sowell ER, Peterson BS, Thompson PM, Welcome SE, Henkenius AL, Toga AW (2003) Mapping cortical change across the human life span. Nat Neurosci 6:309-315. CrossRef Medline

Sowell ER, Thompson PM, Toga AW (2004) Mapping changes in the human cortex throughout the span of life. Neuroscientist 10:372-392. CrossRef Medline

Stiles J, Jernigan TL (2010) The basics of brain development. Neuropsychol Rev 20:327-348. CrossRef Medline

Stüber C, Morawski M, Schäfer A, Labadie C, Wähnert M, Leuze C, Streicher M, Barapatre N, Reimann K, Geyer S, Spemann D, Turner R (2014) Myelin and iron concentration in the human brain: a quantitative study of MRI contrast. Neuroimage 93:95-106. CrossRef Medline

Tustison NJ, Avants BB, Cook PA, Zheng Y, Egan A, Yushkevich PA, Gee JC (2010) N4ITK: improved N3 bias correction. IEEE Trans Med Imaging 29:1310-1320. CrossRef Medline

Tustison NJ, Cook PA, Klein A, Song G, Das SR, Duda JT, Kandel BM, van Strien N, Stone JR, Gee JC, Avants BB (2014) Large-scale evaluation of ANTs and FreeSurfer cortical thickness measurements. Neuroimage 99: 166-179. CrossRef Medline

Tzourio-Mazoyer N, Landeau B, Papathanassiou D, Crivello F, Etard O, Delcroix N, Mazoyer B, Joliot M (2002) Automated anatomical labeling of activations in SPM using a macroscopic anatomical parcellation of the MNI MRI single-subject brain. Neuroimage 15:273-289. CrossRef Medline

Wood SN (2011) Fast stable restricted maximum likelihood and marginal likelihood estimation of semiparametric generalized linear models. J R Stat Soc Series B Stat Methodol 73:3-36. CrossRef

Wood SN (2012) On p-values for smooth components of an extended generalized additive model. Biometrika 100:221-228. CrossRef

Zhang H, Schneider T, Wheeler-Kingshott CA, Alexander DC (2012) NODDI: practical in vivo neurite orientation dispersion and density imaging of the human brain. Neuroimage 61:1000-1016. CrossRef Medline

Zielinski BA, Gennatas ED, Zhou J, Seeley WW (2010) Network-level structural covariance in the developing brain. Proc Natl Acad Sci U S A 107: 18191-18196. CrossRef Medline 